\title{
КОНСТРУИРОВАНИЕ НЕГЛАДКОГО РЕШЕНИЯ ЗАДАЧИ УПРАВЛЕНИЯ ПО БЫСТРОДЕЙСТВИЮ ПРИ НИЗКОМ ПОРЯДКЕ ГЛАДКОСТИ ГРАНИЦЫ ЦЕЛЕВОГО МНОЖЕСТВА ${ }^{1}$
}

\author{
П. Д. Лебедев, А. А. Успенский
}

\begin{abstract}
Для плоской задачи управления по быстродействию с круговой вектограммой скоростей и невыпуклым компактным целевым множеством, имеющим границу с порядком гладкости 1 или 2, разработаны процедуры построения функции оптимального результата. Изучены псевдовершины - характеристические точки границы целевого множества, определяющие характер сингулярности этой функции. Выявлены дифференциальные зависимости для гладких сегментов сингулярного множества, что позволяет их рассматривать и строить в виде дуг интегральных кривых. Найдены необходимые условия существования псевдовершин и получены формулы проекций точек сингулярного множества в окрестности псевдовершин. Предложенные процедуры реализованы в виде вычислительных алгоритмов. Их эффективность проиллюстрирована на примерах численного решения задач управления по быстродействию с различным порядком гладкости границ целевых множеств. Выполнена визуализация результатов.
\end{abstract}

Ключевые слова: задача быстродействия, сингулярное множество, рассеивающая кривая, функция оптимального результата, псевдовершина, множество симметрии.

P. D. Lebedev, A. A. Uspenskii. Construction of a nonsmooth solution in a time-optimal problem with a low order of smoothness of the boundary of the target set.

Procedures for the construction of an optimal result function have been developed for a planar time-optimal control problem with a circular velocity vectorgram and nonconvex compact target set whose boundary has smoothness 1 or 2 . Pseudovertices, which are characteristic points of the boundary of the target set defining the character of the singularity of this function, are studied. Differential dependences for smooth segments of the singular set are revealed, which allows to consider and construct them as arcs of integral curves. The necessary conditions for the existence of pseudovertices are found and formulas for the projections of points of the singular set in neighborhoods of pseudovertices are obtained. The proposed procedures are implemented in the form of computational algorithms. Their efficiency is illustrated by examples of the numerical solution of optimal-time control problems with different orders of smoothness of the boundaries of the target sets. Visualization of the results is performed.

Keywords: time-optimal problem, singular set, dispersing curve, optimal result function, pseudo-vertex, symmetry set.

MSC: 14H20, 35A18, 37G10, 37G40, 51K10

DOI: $10.21538 / 0134-4889-2019-25-1-108-119$

\section{1. Постановка задачи}

Рассмотрим задачу управления по быстродействию, заключающуюся в приведении точки евклидовой плоскости на заданное целевое множество $M \subset \mathbb{R}^{2}$ за минимально возможное время [1]. Полагаем, что движение точки $\mathbf{x}=(x, y)$ задается уравнением

$$
\dot{\mathbf{x}}=\mathbf{v}
$$

где на управление $\mathbf{v}=\left(v_{1}, v_{2}\right)$ наложено ограничение $\|\mathbf{v}\|=\sqrt{v_{1}^{2}+v_{2}^{2}} \leq 1$. Вектограмма скоростей рассматриваемой динамической системы есть круг единичного радиуса с центром в начале координат. В данной постановке задача ранее изучалась авторами с позиции выявления

\footnotetext{
${ }^{1}$ Работа выполнена при финансовой поддержке постановления № 211 Правительства Российской Федерации, контракт № 02.А03.21.0006.
} 
особенностей и построения линий уровня с заданным шагом $[2 ; 3]$. Ранее в качестве целевого множества рассматривались в основном подграфики непрерывных функций $[4 ; 5]$. Однако для практических задач важнее изучать задачи о сближении с компактом, граница которого имеет невыпуклые особенности [6].

$\mathrm{B}$ случае $\mathbf{x} \notin M$ оптимальным управлением $\mathbf{v}$ является вектор единичной нормы, направленный от $\mathbf{x}$ к ближайшей к ней в евклидовой метрике точке $\mathbf{y}$ границы множества $M$. Функция оптимального результата $u(\mathbf{x})=u(x, y)$ (равная минимальному времени, за которое движущаяся точка может достичь $M)$ совпадет с евклидовым расстоянием $\rho(\mathbf{x}, M)=\min \{\|\mathbf{x}-\mathbf{y}\|: \mathbf{y} \in$ $M\}$ от точки $\mathbf{x}=(x, y) \in \mathbb{R}^{2}$ до множества $M$.

В дальнейшем будем рассматривать случай компактного односвязного множества $M$, граница которого Г есть плоская кривая, заданная параметрическим уравнением

$$
\Gamma=\left\{\mathbf{y} \in \mathbb{R}^{2}: \mathbf{y}=\mathbf{y}(t), t \in[0, T]\right\} .
$$

Здесь $T>0$, а отображение $\mathbf{y}:[0, T] \rightarrow \mathbb{R}^{2}$ является непрерывным на отрезке $[0, T]$, дифференцируемым во всех точках интервала $(0, T)$ и дважды дифференцируемым на $(0, T)$ за исключением, может быть, конечного числа точек. Считаем, что (1.2) есть замкнутая регулярная кривая без точек самопересечения, $\mathbf{y}(0)=\mathbf{y}(T)$. При этом производные первого и второго порядка совпадают на концах отрезка $[0, T]$.

Рассматриваемой задаче быстродействия можно поставить в соответствие уравнение Гамильтона - Якоби [7]

$$
\min _{\mathbf{v}:\|\mathbf{v}\| \leq 1}\langle D u(\mathbf{x}), \mathbf{v}\rangle+1=0,
$$

где $D u(\mathbf{x})$ есть градиент функции $u(\mathbf{x})$ в точке $\mathbf{x} ;\langle\cdot, \cdot\rangle$ означает скалярное произведение векторов. Минимаксное решение [8] задачи Дирихле для уравнения (1.3) с краевым условием

$$
\left.u\right|_{\Gamma}=0
$$

совпадает с функцией оптимального результата $u(x, y)$ на множестве $G=\mathbb{R}^{2} \backslash M$ (см. [2, с. 67$]$ ).

$\mathrm{C}$ приведенной задачей связано рассматриваемое в геометрической оптике уравнение эйконала

$$
\left(\frac{\partial u}{\partial x}\right)^{2}+\left(\frac{\partial u}{\partial y}\right)^{2}=1
$$

которое описывает распространение света в изотропной среде. Задача Дирихле для уравнения (1.5) с краевым условием (1.4) имеет обобщенное (фундаментальное) решение, равное $u_{k}(\mathbf{x})=-\rho(\mathbf{x}, M)$ (подробнее см. [9]). Его смысл - это величина оптического пути между точкой $\mathbf{x}$ и множеством $M$, имеющим границу $Г$.

В рамках данной статьи описывается решение указанных задач на базе построения сингулярных множеств, на которых функция $u(x, y)$ теряет гладкость. Их отыскание позволяет конструировать как линии уровня функции $u(x, y)$, так и ее график в виде поверхности в трехмерном пространстве. Показано, что сингулярные множества могут быть найдены посредством решения краевых задач Коши для дифференциального уравнения, связывающего координаты точек границы множества $M$.

\section{2. Сингулярные множества в задаче быстродействия}

В случае выпуклого множества $M$ функция $u(\mathbf{x})=\rho(\mathbf{x}, M)$ выпукла на всей плоскости $\mathbb{R}^{2}$ и дифференцируема на множестве $G$ (см. [10, гл. II, § 8]). Если же множество $M$ не выпукло, то у $u(\mathbf{x})$ возникают сингулярные множества, на которых $u(\mathbf{x})$ теряет гладкость.

О п р е д е л е н и е 1 . Множеством $\Omega_{M}(\mathbf{x})$ проекций точки $\mathbf{x}$ на множество $M$ назовем объединение всех точек $\mathbf{y} \in M$, ближайших в евклидовой метрике к $\mathbf{x}$. 
О п р е д е л е н и е 2. Биссектрисой $L(M)$ замкнутого множества $M \subset \mathbb{R}^{2}$ назовем [3] множество всех точек, для которых множество $\Omega_{M}(\mathbf{x})$ состоит из двух или более элементов:

$$
L(M)=\left\{\mathbf{x} \in \mathbb{R}^{2}: \exists \mathbf{y}_{1} \in \Omega_{M}(\mathbf{x}), \mathbf{y}_{2} \in \Omega_{M}(\mathbf{x})\left(\mathbf{y}_{1} \neq \mathbf{y}_{2}\right)\right\} .
$$

Биссектриса множества является частным случаем множеств симметрии [11;12], используемых, например, при описании волновых фронтов и каустик [13]. Современные исследования в данных областях часто привлекают к рассмотрению фигуры с границей $\Gamma$, имеющей особенности (особые точки, разрывы кривизны), при этом выявляется связь между геометрией Г и структурой множеств симметрии (см. $[14 ; 15])$.

В качестве другого представителя множеств симметрии (лежащего не вовне множества $M$, а внутри него) можно указать так называемый скелет множества, активно используемый Л. М. Местецким в задачах распознавания образов [16, с. 32]. По определению скелет $\mathbf{S}(M)$ множества $M \subset \mathbb{R}^{2}$ есть геометрическое место центров кругов $O(\mathbf{x}, r)$ радиуса $r \in[0,+\infty)$ с центром в точке $\mathbf{x}$, для которых выполняются следующие включения:

$$
\begin{gathered}
O(\mathbf{x}, r) \subseteq M ; \\
\forall \varepsilon>0 O(\mathbf{x}, r+\varepsilon) \cap\left(\mathbb{R}^{2} \backslash M\right) \neq \varnothing .
\end{gathered}
$$

Справедливо равенство

$$
\mathbf{S}(M)=\operatorname{cl} L\left(\operatorname{cl}\left(\mathbb{R}^{2} \backslash M\right)\right),
$$

связывающее его с биссектрисой множества. Здесь и далее $\mathrm{cl} X$ означает замыкание множества $X$.

Согласно классификации Р. Айзекса $L(M)$ - рассеивающая линия в задаче быстродействия для динамической системы (1.1): из каждой ее точки исходит более одной оптимальной траектории $[7$, с. 196]. Этим вызвано нарушение на $L(M)$ гладкости функции оптимального результата, характерное для сингулярных кривых.

О п р е д е л е н и е 3 . Точка $\mathbf{x} \in L(M)$ биссектрисы $L(M)$ называется порожденной парой точек $\left\{\mathbf{y}_{1}, \mathbf{y}_{2}\right\} \in \partial M$, если $\left\{\mathbf{y}_{1}, \mathbf{y}_{2}\right\} \subseteq \Omega_{M}(\mathbf{x})$ и $\mathbf{y}_{1} \neq \mathbf{y}_{2}$. При этом $\mathbf{y}_{1}$ и $\mathbf{y}_{2}$ называются $\alpha$-симметричными точками [6].

При практической реализации построения гладких участков $L(M)$ естественно найти зависимость $t_{2}=t_{2}\left(t_{1}\right), t_{1} \in \mathbb{R}$, связывающую параметры, задающие $\alpha$-симметричные точки. Однако на практике это трудно реализовать в виде численного алгоритма.

При построении рассеивающих линий в задаче быстродействия лучше всего получить формулы, которые в наименьшей степени зависят от параметризации кривой Г. Одной из основных характеристик плоской кривой является ее кривизна. Для плоской кривой (1.2) значение кривизны $k=k(t)$ в точке $\mathbf{y}(t)$ вычисляется следующим образом:

$$
k(t)=\frac{\mathbf{y}^{\prime \prime}(t) \wedge \mathbf{y}^{\prime}(t)}{\left\|\mathbf{y}^{\prime}(t)\right\|^{3 / 2}} .
$$

Здесь $\left(a_{1}, a_{2}\right) \wedge\left(b_{1}, b_{2}\right)=a_{1} b_{2}-a_{2} b_{1}$. Полагаем без ограничения общности, что при изменении параметра $t$ от 0 до $T$ вектор $\mathbf{y}(t)$ делает оборот вокруг внутренних точек множества $M$ против часовой стрелки. С кривизной тесно связаны понятия радиуса кривизны $r(t)=1 / k(t)$ и центра кривизны $\mathbf{c}(t)$ - точки, расположенной на нормали к $Г$ в точке $\mathbf{y}(t)$ на расстоянии $r(t)$ от нее в направлении, противоположном локальной выпуклости (см. [17, гл. III, §25]).

Теорема 1 (О структуре гладкого участка биссектрисы). Пусть точка биссектрисы $\mathbf{x} \in$ $L(M)$ имеет ровно две проекиии $\mathbf{y}_{1}=\mathbf{y}\left(t_{1}\right)$ и $\mathbf{y}_{2}=\mathbf{y}\left(t_{2}\right)$ на компактное односвязное множество $M$, при этом $\mathbf{x} \neq\left(\mathbf{y}_{1}+\mathbf{y}_{2}\right) / 2$. Тогда для параметров $t_{1}, t_{2}$, задающих координать $\alpha$-симметричных точек, справедливо дифференииальное соотночение

$$
\frac{d t_{2}}{d t_{1}}=-\frac{k\left(t_{1}\right) r+1}{k\left(t_{2}\right) r+1} \cdot \frac{\left\|\mathbf{y}^{\prime}\left(t_{1}\right)\right\|}{\left\|\mathbf{y}^{\prime}\left(t_{2}\right)\right\|}
$$

где $r=\rho(\mathbf{x}, M)$. 
Доказательство теоремы приведено в [6, с. 186]. Заметим, что под условия теоремы попадают только те проекции точек биссектрисы, в которых определены и касательная, и кривизна границы множества $M$. Из теоремы 1 вытекает, что если множество проекций точки $\mathbf{x}^{*} \in L(M)$ состоит ровно из двух элементов, $\Omega_{M}\left(\mathbf{x}^{*}\right)=\left\{\mathbf{y}_{1}, \mathbf{y}_{2}\right\}$, то к $L(M)$ в $\mathbf{x}^{*}$ определена касательная П, совпадающая со срединным перпендикуляром к отрезку $\left[\mathbf{y}_{1}, \mathbf{y}_{2}\right]$ (что было ранее доказано в [4, c. 192]).

Важную роль при построении сингулярного множества $M$ играют некоторые характеристические точки - псевдовершины.

О п р е д е л е н и е 4 . Будем называть точку $\mathbf{y}_{0}=\mathbf{y}\left(t_{0}\right)$ псевдовершиной [3] множества $M$, если существует последовательность $\left\{\left(\overline{\mathbf{y}}_{n}, \widetilde{\mathbf{y}}_{n}\right)\right\}_{n=1}^{\infty}$ пар $\alpha$-симметричных точек, для которой имеет место предел:

$$
\lim _{n \rightarrow \infty}\left(\overline{\mathbf{y}}_{n}, \widetilde{\mathbf{y}}_{n}\right)=\left(\mathbf{y}_{0}, \mathbf{y}_{0}\right) .
$$

О п р е д е л е н и е 5 . Пусть $\mathbf{y}_{0}$ - псевдовершина множества $M$ с биссектрисой $L(M)$. Будем говорить, что точка $\widehat{\mathbf{x}}$ есть крайняя точка биссектрисы [8], соответствующая псевдовершине $\mathbf{y}_{0}$, если существуют последовательности $\left\{\left(\overline{\mathbf{y}}_{n}, \widetilde{\mathbf{y}}_{n}\right)\right\}_{n=1}^{\infty} \subset \partial M$ и $\left\{\mathbf{x}_{n}\right\}_{n=1}^{\infty} \subset L(M)$, для которых выполняются условия

$$
\begin{aligned}
& \text { 1) } \lim _{n \rightarrow \infty}\left(\overline{\mathbf{y}}_{n}, \widetilde{\mathbf{y}}_{n}\right)=\left(\mathbf{y}_{0}, \mathbf{y}_{0}\right) ; \\
& \text { 2) } \lim _{n \rightarrow \infty} \mathbf{x}_{n}=\widehat{\mathbf{x}} ; \\
& \text { 3) } \forall n \in \mathbb{N}\left(\overline{\mathbf{y}}_{n}, \widetilde{\mathbf{y}}_{n}\right) \subset \Omega_{M}\left(\mathbf{x}_{n}\right) .
\end{aligned}
$$

Легко показать, что отображение $\mathbf{x} \mapsto \Omega_{M}(\mathbf{x})$ является полунепрерывным сверху по включению. Рассмотрим сходящиеся последовательности точек $\left\{\mathbf{x}_{n}\right\}_{n=1}^{\infty} \subset L(M)$ и $\left\{\mathbf{y}_{n}\right\}_{n=1}^{\infty} \subset \partial M$, такие что

$$
\lim _{n \rightarrow \infty} \mathbf{x}_{n}=\mathbf{x}_{0}, \quad \lim _{n \rightarrow \infty} \mathbf{y}_{n}=\mathbf{y}_{0}, \quad \forall n \in \mathbb{N} \mathbf{y}_{n} \in \Omega_{M}\left(\mathbf{x}_{n}\right) .
$$

Покажем, что имеет место

$$
\mathbf{y}_{0} \in \Omega_{M}\left(\mathbf{x}_{0}\right) \text {. }
$$

Допустим, (2.4) не выполняется. Тогда найдется точка $\mathbf{y}^{*} \in M$, такая что $\left\|\mathbf{x}_{0}-\mathbf{y}^{*}\right\|<\left\|\mathbf{x}_{0}-\mathbf{y}_{0}\right\|$. Рассмотрим круг $O\left(\mathbf{x}_{0}, \Delta r\right)$, где $\Delta r=\left(\left\|\mathbf{x}_{0}-\mathbf{y}_{0}\right\|-\left\|\mathbf{x}_{0}-\mathbf{y}^{*}\right\|\right) / 4$. В нем должны находиться все точки $\mathbf{x}_{n}$, начиная с некоторого номера $n^{*}$. При этом согласно неравенству треугольника для них выполняются неравенства

$$
\left\|\mathbf{x}_{n}-\mathbf{y}^{*}\right\| \leqslant\left\|\mathbf{x}_{n}-\mathbf{y}_{0}\right\|+4 \Delta r-2 \Delta r=\left\|\mathbf{x}_{n}-\mathbf{y}_{0}\right\|+2 \Delta r
$$

Значит, начиная с некоторого номера, точки $\mathbf{y}_{n}$ не могут лежать в окрестности точки $\mathbf{y}_{n}$ радиуса $2 \Delta r>0$. Получилось противоречие.

Следовательно, справедливо включение относительно псевдовершины и соответствующей ей крайней точки биссектрисы

$$
\mathbf{y}_{0} \in \Omega_{M}(\widehat{\mathbf{x}}) .
$$

Теорема 2 (О характере псевдовершины второго порядка гладкости). Пусть точка $\mathbf{y}_{0}=$ $\mathbf{y}\left(t_{0}\right) \in \Gamma$, в которой определена кривизна $k\left(t_{0}\right)$, является псевдовершиной множества $M$ и ей соответствует крайняя точка $\widehat{\mathbf{x}}$ биссектрисы $L(M)$. Тогда справедливы три утверждения.

1) Модуль $|k(t)|$ кривизны $\Gamma$ достигает локального максимума при $t=t_{0}$.

2) Точка $\widehat{\mathbf{x}}$ есть центр кривизны $\mathbf{c}\left(t_{0}\right)$ кривой $\Gamma$ в $\mathbf{y}\left(t_{0}\right)$.

3) Выполняется предельное соотношение

$$
\lim _{t_{1} \rightarrow t_{0}-0} \frac{t_{2}\left(t_{1}\right)-t_{0}}{t_{1}-t_{0}}=-1
$$

для параметров $t_{1}, t_{2}$, задающих координаты $\alpha$-симметричных точек (в окрестности $t_{0}$ ). 
Доказательства утверждений 1) и 2) вытекают из теоремы 1 работы [3] (в ней не введено определение крайней точки биссектрисы, но по сути ее роль играет точка $V$ - предел пересечения нормалей к $\Gamma$ в $\alpha$-симметричных точках при стремлении их к псевдовершине), а утверждения 3$)$ - из [5, лемма 1$]$.

Теоремы 1 и 2 позволяют находить координаты $\alpha$-симметричных точек как решение задачи Коши для дифференциального уравнения первого порядка:

$$
\left\{\begin{array}{l}
\frac{d t_{2}}{d t_{1}}=g\left(t_{1}, t_{2}\right) \\
t_{2}\left(x_{0}\right)=t_{0}
\end{array}\right.
$$

где

$$
g\left(x_{1}, x_{2}\right)=\left\{\begin{array}{l}
-\frac{k\left(t_{1}\right) r\left(t_{1}, t_{2}\right)+1}{k\left(t_{2}\right) r\left(t_{1}, t_{2}\right)+1} \cdot \frac{\left\|\mathbf{y}^{\prime}\left(t_{1}\right)\right\|}{\left\|\mathbf{y}^{\prime}\left(t_{2}\right)\right\|}, \quad t_{1}<t_{0}<t_{2} \\
-1, t_{1}=t_{2}=t_{0}
\end{array}\right.
$$

здесь кривизна $k\left(t_{i}\right), i=1,2$ определяется по формуле $(2.2)$;

$$
r\left(t_{1}, t_{2}\right)=\frac{\left\|\mathbf{y}\left(t_{2}\right)-\mathbf{y}\left(t_{1}\right)\right\|}{2 \sin \left(\angle\left(\mathbf{y}\left(t_{2}\right), \mathbf{y}^{\prime}\left(t_{1}\right)\right) / 2\right)}
$$

есть расстояние от точек $\mathbf{y}\left(t_{1}\right)$ и $\mathbf{y}\left(t_{1}\right)$ до точки пересечения перпендикуляров, построенных к ним к кривой $\Gamma, \angle(\mathbf{a}, \mathbf{b})$ - угол между векторами $\mathbf{a}$ и $\mathbf{b}$. При этом параметр $t_{1}$ меняется на некотором отрезке $\left[t_{0}-\varepsilon_{1}, t_{0}\right], \varepsilon_{1}>0$, а параметр $t_{2}$ - на некотором отрезке $\left[t_{0}+\varepsilon_{2}, t_{0}\right], \varepsilon_{2}>0$, т. е. $t_{2}>t_{1}$ (за исключением точки $t_{1}=t_{2}=t_{0}$, которая входит в решение задачи $(2.7)$, но задает координаты псевдовершины, а не проекций точек биссектрисы).

Теорема 3 (О характере псевдовершины при разрыве гладкости по второму порядку).

Пусть точка $\mathbf{y}_{0}=\mathbf{y}\left(t_{0}\right) \in \Gamma$ является псевдовершиной множества $M$ и ей соответствует крайняя точка $\widehat{\mathbf{x}}$ биссектрисы $L(M)$, при этом определены односторонние значения кривизны $k\left(t_{0}-0\right)$ и $k\left(t_{0}+0\right)$ одного знака и $k\left(t_{0}-0\right) \neq k\left(t_{0}+0\right)$.

Тогда если $\left|k\left(t_{0}-0\right)\right|>\left|k\left(t_{0}+0\right)\right|$, то справедливы следующие утверждения:

A1) Модуль кривизны $|k(t)|$ возрастает на некоторой левой полуокрестности $\left(t_{0}-\varepsilon, t_{0}\right)$, $\varepsilon>0$ точки $t=t_{0}$.

А2) Точка $\widehat{\mathbf{x}}$ есть предел $\mathbf{c}\left(t_{0}-0\right)$ чентров кривизны кривой $\Gamma$ в точках $\mathbf{y}(t)$ при $t \rightarrow t_{0}-0$.

А3) Выполняется предельное соотношение

$$
\lim _{t_{1} \rightarrow t_{0}-0} \frac{t_{2}\left(t_{1}\right)-t_{0}}{t_{1}-t_{0}}=0 .
$$

Если $\left|k\left(t_{0}-0\right)\right|<\left|k\left(t_{0}+0\right)\right|$, то справедливы следующие утверждения:

B1) Норма кривизны $|k(t)|$ возрастает на некоторой правой полуокрестности $\left(t_{0}, t_{0}+\right.$ $\varepsilon), \varepsilon>0$, точки $t=t_{0}$.

В2) Точка $\widehat{\mathbf{x}}$ есть предел $\mathbf{c}\left(t_{0}+0\right)$ центров кривизны кривой $\Gamma$ в точках $\mathbf{y}(t)$ nри $t \rightarrow t_{0}+0$.

В3) Выполняется предельное соотношение

$$
\lim _{t_{1} \rightarrow t_{0}-0} \frac{t_{1}-t_{0}}{t_{2}\left(t_{1}\right)-t_{0}}=0
$$

Д о к а з а т е л ь с т в о. Рассмотрим случай $\left|k\left(t_{0}-0\right)\right|>\left|k\left(t_{0}+0\right)\right|$. Без ограничения общности полагаем, что точка $\mathbf{y}_{0}$ совпадает с началом координат, касательная к кривой $Г$ в ней совпадает с осью абсцисс, а направление выпуклости Г в ней совпадает с положительным направлением оси ординат. Условия на границу $\Gamma(1.2)$ множества $M$ позволяют представить ее в некоторой окрестности псевдовершины в виде графика gr $y(x)$ функции $y=f(x)$ с областью определения $(-\varepsilon, \varepsilon), \varepsilon>0$. 
Рассмотрим абсциссы $x_{1}$ и $x_{2}$ проекций точек биссектрисы, лежащих в окрестности псевдовершины, и точку $\left(x^{*}, y^{*}\right)$ пересечения нормалей к $\operatorname{gr} y(x)$ в них. По построению для значений имеет место

$$
\begin{gathered}
f(0)=0 \\
f^{\prime}(0)=0 \\
f^{\prime \prime}(-0)=\left|k\left(t_{0}-0\right)\right|, \quad f^{\prime \prime}(+0)=\left|k\left(t_{0}+0\right)\right| .
\end{gathered}
$$

Обозначим ординаты точек как $y_{i}=f\left(x_{i}\right), i=1,2$, а значения производных функции $y=$ $f(x)$ в них как $y_{i}^{\prime}=f^{\prime}\left(x_{i}\right), y_{i}^{\prime \prime}=f^{\prime \prime}\left(x_{i}\right), i=1,2$. Получаем выражения для координат точек биссектрисы:

$$
\begin{aligned}
x^{*} & =\frac{\left(x_{1}+y_{1} y_{1}^{\prime}\right) y_{2}^{\prime}-\left(x_{2}+y_{2} y_{2}^{\prime}\right) y_{1}^{\prime}}{y_{2}^{\prime}-y_{1}^{\prime}}, \\
y^{*} & =\frac{-\left(x_{1}+y_{1} y_{1}^{\prime}\right)+\left(x_{2}+y_{2} y_{2}^{\prime}\right)}{y_{2}^{\prime}-y_{1}^{\prime}} .
\end{aligned}
$$

В некоторой окрестности точки 0 можно считать $x_{2}$ функцией от $x_{1}$, поскольку между абсциссами проекций точек биссектрисы в ней существует взаимно однозначное соответствие. Покажем, что выполняется соотношение

$$
\lim _{x_{1} \rightarrow-0} \frac{x_{2}}{x_{1}}=0
$$

Допустим, что (2.15) не выполняется. Тогда найдутся такие последовательности $\left\{\bar{x}^{(i)}\right\}_{i=1}^{\infty}$ и $\left\{\widetilde{x}^{(i)}\right\}_{i=1}^{\infty}$, что

$$
\begin{gathered}
\forall i \in \mathbb{N} \bar{x}^{(i)}<0, \quad \widetilde{x}^{(i)}>0 ; \\
\lim _{i \rightarrow \infty} \bar{x}^{(i)}=\lim _{i \rightarrow \infty} \widetilde{x}^{(i)}=0, \\
\lim _{i \rightarrow \infty} \frac{\widetilde{x}^{(i)}}{\bar{x}^{(i)}}=c^{*}<0 .
\end{gathered}
$$

Условия (2.16)-(2.18) означают, что элементы последовательностей могут быть представлены в виде

$$
\bar{x}^{(i)}=\kappa_{1} t^{(i)}+o\left(t^{(i)}\right), \widetilde{x}^{(i)}=\kappa_{2} t^{(i)}+o\left(t^{(i)}\right),
$$

где

$$
\forall i \in \mathbb{N} t^{(i)}>0, \quad \lim _{i \rightarrow \infty} t^{(i)}=0, \quad \kappa_{1} \leq 0, \quad \kappa_{2}>0,
$$

$o(t)$ - бесконечно малая функция более высокого порядка, чем $t$ (т. е. $\left.\lim _{t \rightarrow 0} t^{-1} o(t)=0\right)$.

Представим разложение значений функции $f(x)$ и ее производной $f^{\prime}(x)$ по формуле Тейлора в окрестности точки $x=0$ с учетом равенств $(2.10)-(2.12)$ :

$$
\begin{gathered}
f\left(\bar{x}^{(i)}\right)=f(0)+f^{\prime}(0) \bar{x}^{(i)}+o\left(\bar{x}^{(i)}\right)=o\left(\bar{x}^{(i)}\right)=o\left(t^{(i)}\right), \\
f\left(\widetilde{x}^{(i)}\right)=f(0)+f^{\prime}(0) \widetilde{x}^{(i)}+o\left(\widetilde{x}^{(i)}\right)=o\left(\widetilde{x}^{(i)}\right)=o\left(t^{(i)}\right), \\
f^{\prime}\left(\bar{x}^{(i)}\right)=f^{\prime}(0)+f^{\prime \prime}(-0) \bar{x}^{(i)}+o\left(\bar{x}^{(i)}\right)=f^{\prime \prime}(-0)\left(\kappa_{1} t^{(i)}+o\left(t^{(i)}\right)\right)+o\left(t^{(i)}\right)=f^{\prime \prime}(-0) \kappa_{1} t^{(i)}+o\left(t^{(i)}\right), \\
f^{\prime}\left(\widetilde{x}^{(i)}\right)=f^{\prime}(0)+f^{\prime \prime}(+0) \widetilde{x}^{(i)}+o\left(\widetilde{x}^{(i)}\right)=f^{\prime \prime}(+0)\left(\kappa_{2} t^{(i)}+o\left(t^{(i)}\right)\right)+o\left(t^{(i)}\right)=f^{\prime \prime}(+0) \kappa_{2} t^{(i)}+o\left(t^{(i)}\right) .
\end{gathered}
$$

Тогда, подставив значения (2.19) в формулы 2.13, (2.14), можно получить выражения, задающие координаты $(\widehat{x}, \widehat{y})$ крайней точки биссектрисы:

$$
\widehat{x}=\lim _{i \rightarrow \infty}\left(\frac{\left(\bar{x}^{(i)}+f\left(\bar{x}^{(i)}\right) f^{\prime}\left(\bar{x}^{(i)}\right)\right) f^{\prime}\left(\widetilde{x}^{(i)}\right)}{f^{\prime}\left(\widetilde{x}^{(i)}\right)-f^{\prime}\left(\bar{x}^{(i)}\right)}-\frac{\left(\widetilde{x}^{(i)}+f\left(\widetilde{x}^{(i)}\right) f^{\prime}\left(\widetilde{x}^{(i)}\right)\right) f^{\prime}\left(\bar{x}^{(i)}\right)}{f^{\prime}\left(\widetilde{x}^{(i)}\right)-f^{\prime}\left(\bar{x}^{(i)}\right)}\right)
$$




$$
\begin{gathered}
=\lim _{i \rightarrow \infty}\left(\frac{\left(\kappa_{1} t^{(i)}+o\left(t^{(i)}\right)+o\left(t^{(i)}\right)\right)\left(f^{\prime \prime}(+0) \kappa_{2} t^{(i)}+o\left(t^{(i)}\right)\right)}{f^{\prime \prime}(+0) \kappa_{2} t^{(i)}+o\left(t^{(i)}\right)-f^{\prime \prime}(-0) \kappa_{1} t^{(i)}-o\left(t^{(i)}\right)}\right. \\
\left.-\frac{\left(\kappa_{2} t^{(i)}+o\left(t^{(i)}\right)+o\left(t^{(i)}\right)\right)\left(f^{\prime \prime}(-0) \kappa_{1} t^{(i)}+o\left(t^{(i)}\right)\right)}{f^{\prime \prime}(+0) \kappa_{2} t^{(i)}+o\left(t^{(i)}\right)-f^{\prime \prime}(-0) \kappa_{1} t^{(i)}-o\left(t^{(i)}\right)}\right) \\
=\lim _{i \rightarrow \infty} \frac{o\left(t^{(i)}\right)}{\left(f^{\prime \prime}(+0) \kappa_{2}-f^{\prime \prime}(-0) \kappa_{1}\right) t^{(i)}+o\left(t^{(i)}\right)}=0, \\
\widehat{y}=\lim _{i \rightarrow \infty} \frac{-\left(\bar{x}^{(i)}+f\left(\bar{x}^{(i)}\right) f^{\prime}\left(\bar{x}^{(i)}\right)\right)+\left(\widetilde{x}^{(i)}+f\left(\widetilde{x}^{(i)}\right) f^{\prime}\left(\widetilde{x}^{(i)}\right)\right)}{f^{\prime}\left(\widetilde{x}^{(i)}\right)-f^{\prime}\left(\bar{x}^{(i)}\right)} \\
=\lim _{i \rightarrow \infty} \frac{\left(\kappa_{2}-\kappa_{1}\right) t^{(i)}+o\left(t^{(i)}\right)}{\left(f^{\prime \prime}(+0) \kappa_{2}-f^{\prime \prime}(-0) \kappa_{1}\right) t^{(i)}+o\left(t^{(i)}\right)}=\frac{\kappa_{2}-\kappa_{1}}{f^{\prime \prime}(+0) \kappa_{2}-f^{\prime \prime}(-0) \kappa_{1}} .
\end{gathered}
$$

Если $\kappa_{2}>0$, то значение (2.21) можно оценить:

$$
\begin{gathered}
\widehat{y}=\left(\frac{\kappa_{2}}{\kappa_{2}-\kappa_{1}} \frac{1}{f^{\prime \prime}(+0)}+\frac{-\kappa_{1}}{\kappa_{2}-\kappa_{1}} \frac{1}{f^{\prime \prime}(-0)}\right)^{-1}>\left(\frac{\kappa_{2}}{\kappa_{2}-\kappa_{1}} \frac{1}{f^{\prime \prime}(-0)}+\frac{-\kappa_{1}}{\kappa_{2}-\kappa_{1}} \frac{1}{f^{\prime \prime}(-0)}\right)^{-1} \\
=\frac{\kappa_{2}-\kappa_{1}}{\kappa_{2}-\kappa_{1}} \frac{1}{f^{\prime \prime}(-0)}=\left|k\left(t_{0}-0\right)\right|^{-1},
\end{gathered}
$$

т. е. ордината крайней точки биссектрисы больше, чем предельное значение радиуса кривизны слева в псевдовершине. С учетом $(2.20)$ это означает, что крайняя точка $(\widehat{x}, \widehat{y})$ лежит на нормали к биссектрисе в псевдовершине на расстоянии, равном (2.21), превышающем предельное значение радиуса кривизны $r\left(t_{0}-0\right)$ слева, с той же стороны от $\mathbf{y}_{0}$, что и предельное положение центра кривизны. Однако если рассмотреть предельную соприкасающуюся окружность [17] слева в точке $x=0$, то она будет иметь радиус $1 / f^{\prime \prime}(-0)$, следовательно, она будет заходить внутрь круга $O\left(\widehat{\mathbf{x}},\left\|\mathbf{y}_{0}-\widehat{\mathbf{x}}\right\|\right)$ (здесь и далее будем обозначать $O(\mathbf{x}, r) \triangleq\left\{\mathbf{y} \in \mathbb{R}^{2}:\|\mathbf{y}-\mathbf{x}\| \leqslant r\right\}-$ круг радиуса $r$ с центром в точке $x$ ). Поскольку кривая имеет с соприкасающейся окружностью касание второго порядка, то в некоторой малой окрестности псевдовершины найдутся точки кривой $\Gamma$, которые лежат ближе к крайней точке $\widehat{\mathbf{x}}$, чем $\mathbf{y}_{0}$. Получилось противоречие со свойством (2.5) псевдовершины и соответствующей ей крайней точки. Таким образом, (2.15) выполняется.

Для приращений длин $s_{1}(t), s_{2}(t)$ дуги кривой $\Gamma$, отсчитываемой от псевдовершины $\mathbf{y}_{0}$ до точек $\mathbf{y}\left(t_{1}\right)$ и $\mathbf{y}\left(t_{2}\right)$, можно записать соотношение

$$
\left.\frac{d s_{2}}{d s_{1}}\right|_{t=t_{0}}=\left.\frac{d t_{2}}{d t_{1}}\right|_{t=t_{0}} \frac{\left\|\mathbf{y}^{\prime}\left(t_{0}-0\right)\right\|}{\left\|\mathbf{y}^{\prime}\left(t_{0}+0\right)\right\|}=\left.\frac{d t_{2}}{d t_{1}}\right|_{t=t_{0}} \frac{\left\|\mathbf{y}^{\prime}\left(t_{0}\right)\right\|}{\left\|\mathbf{y}^{\prime}\left(t_{0}\right)\right\|}=\left.\frac{d t_{2}}{d t_{1}}\right|_{t=t_{0}} .
$$

С другой стороны,

$$
\left.\frac{d s_{2}}{d s_{1}}\right|_{t=t_{0}}=\left.\frac{d x_{2} \sqrt{1+y^{\prime}(0)^{2}}}{d x_{1} \sqrt{1+y^{\prime}(0)^{2}}}\right|_{t=t_{0}}=\left.\frac{d x_{2}}{d x_{1}}\right|_{t=t_{0}} .
$$

Значит, имеет место и равенство $\left.\frac{d t_{2}}{d t_{1}}\right|_{t=t_{0}}=\left.\frac{d x_{2}}{d x_{1}}\right|_{t=t_{0}}$, из которого с учетом (2.15) вытекает предельное соотношение (2.8).

Поскольку $x_{2}=o\left(x_{1}\right)$, то координаты крайней точки биссектрисы определяются как

$$
\begin{gathered}
\widehat{x}=\lim _{x_{1} \rightarrow-0} \frac{\left(x_{1}+y_{1} y_{1}^{\prime}\right) y_{2}^{\prime}-\left(x_{2}+y_{2} y_{2}^{\prime}\right) y_{1}^{\prime}}{y_{2}^{\prime}-y_{1}^{\prime}}=\lim _{x_{1} \rightarrow-0} \frac{o\left(x_{1}\right)}{o\left(x_{1}\right)-y^{\prime \prime}(-0) x_{1}+o\left(x_{1}\right)}=0, \\
\widehat{y}=\lim _{x_{1} \rightarrow-0} \frac{-\left(x_{1}+y_{1} y_{1}^{\prime}\right)+\left(x_{2}+y_{2} y_{2}^{\prime}\right)}{y_{2}^{\prime}-y_{1}^{\prime}}=\lim _{x_{1} \rightarrow-0} \frac{-x_{1}+o\left(x_{1}\right)}{o\left(x_{1}\right)-y^{\prime \prime}(-0) x_{1}+o\left(x_{1}\right)}
\end{gathered}
$$




$$
=\lim _{x_{1} \rightarrow-0} \frac{-x_{1}}{-y^{\prime \prime}(-0) x_{1}}=\frac{1}{y^{\prime \prime}(-0)} .
$$

Значит, точка $(\widehat{x}, \widehat{y})$ совпадает с предельным положением $\left(0, y^{\prime \prime}(-0)^{-1}\right)$ центра кривизны графика функции $f(x)$ слева в точке $x=0$.

Отсюда следует, что выполняется и утверждение А1. Если кривизна графика функции $f(x)$ убывает на некотором конечном отрезке $[-\varepsilon, 0]$, то график заходит внутрь предельного положения соприкасающейся окружности $\partial O\left(\widehat{\mathbf{x}},\left\|\mathbf{y}_{0}-\widehat{\mathbf{x}}\right\|\right)$. В этом случае на $Г$ также найдутся точки на расстоянии, меньшем $\left\|\mathbf{y}_{0}-\widehat{\mathbf{x}}\right\|$, от крайней точки биссектрисы, что противоречит (2.5).

Доказательство утверждений В1-В3 аналогично доказательству А1-A3.

Заметим, что в данной статье не рассматриваются псевдовершины, в которой предельные значения кривизны Г слева и справа имеют различный знак. Данный случай требует отдельного рассмотрения, поскольку в нем возможны ситуации, когда одной псевдовершине $\mathbf{y}_{0}$ соответствуют две различные крайние точки бисссектрисы, находящиеся на разных направлениях нормали к кривой $Г$ в $\mathbf{y}_{0}$.

Теоремы 1 и 3 дают возможность находить координаты $\alpha$-симметричных в окрестности псевдовершин с разрывом кривизны посредством решения задачи Коши.

В случае если выполняется $\left|k\left(t_{0}-0\right)\right|>\left|k\left(t_{0}+0\right)\right|$, то задача Коши для дифференциального уравнения имеет вид

$$
\left\{\begin{array}{l}
\frac{d t_{2}}{d t_{1}}=g^{(1)}\left(t_{1}, t_{2}\right) \\
t_{2}\left(x_{0}\right)=t_{0}
\end{array}\right.
$$

где

$$
g^{(1)}\left(t_{1}, t_{2}\right)=\left\{\begin{array}{cc}
-\frac{k\left(t_{1}\right) r\left(t_{1}, t_{2}\right)+1}{k\left(t_{2}\right) r\left(t_{1}, t_{2}\right)+1} \cdot \frac{\left\|\mathbf{y}^{\prime}\left(t_{1}\right)\right\|}{\left\|\mathbf{y}^{\prime}\left(t_{2}\right)\right\|}, & t_{1}<t_{0}<t_{2}, \\
0, & t_{1}=t_{2}=t_{0},
\end{array}\right.
$$

Если же выполняется $\left|k\left(t_{0}-0\right)\right|<\left|k\left(t_{0}+0\right)\right|$, то задача принимает вид, несколько отличающийся от предыдущих, а именно

$$
\left\{\begin{array}{l}
\frac{d t_{1}}{d t_{2}}=g^{(2)}\left(t_{1}, t_{2}\right) \\
t_{2}\left(t_{0}\right)=t_{0}
\end{array}\right.
$$

где

$$
g^{(2)}\left(t_{1}, t_{2}\right)=\left\{\begin{array}{cc}
-\frac{k\left(t_{2}\right) r\left(t_{1}, t_{2}\right)+1}{k\left(t_{1}\right) r\left(t_{1}, t_{2}\right)+1} \cdot \frac{\left\|\mathbf{y}^{\prime}\left(t_{2}\right)\right\|}{\left\|\mathbf{y}^{\prime}\left(t_{1}\right)\right\|}, & t_{1}<t_{0}<t_{2}, \\
0, & t_{1}=t_{2}=t_{0} .
\end{array}\right.
$$

Ее особенностью служит то, что рассматривается производная переменной $t_{1}$ по $t_{2}$. Это позволяет доопределить производную конечным односторонним пределом при $t_{1}=t_{2}=t_{0}$.

Пределы изменения переменных в задачах (2.22) и (2.23) такие же, как и в задаче (2.7).

Заметим, что биссектриса не является всюду гладкой кривой в общем случае, а значит, и зависимости между $t_{1}$ и $t_{2}$ могут описываться как решения задач Коши только локально. Если у $L(M)$ найдена точка бифуркации $\overline{\mathbf{x}}$, то для каждой из сходящихся в ней веток надо выписывать свое уравнение и начальные условия. Если одна из гладких ветвей $L^{*} \subset L(M)$ начинается в $\overline{\mathbf{x}}$ и не содержит крайнюю точку, то для координат ее проекций начальное условие можно найти, исходя из предельного положения проекций точек $\mathbf{x} \in L^{*}$ при их стремлении K $\overline{\mathbf{x}}$.

\section{3. Примеры решения задач}

Авторами разработан программный комплекс [19] средствами пакета MATLAB. Он позволяет выполнять построение сингулярных множеств, волновых фронтов и функции оптимального результата в задаче быстродействия с круговой вектограммой скоростей и невыпуклым 


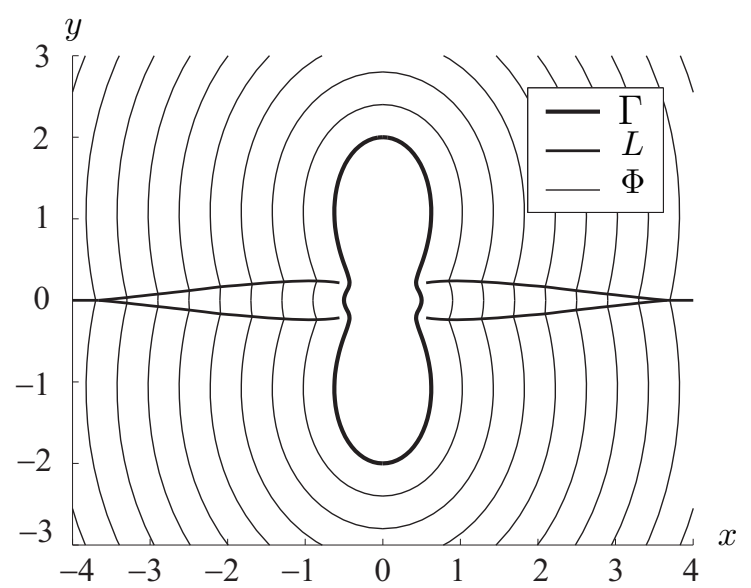

Рис. 1. Линии уровня решения задачи (1.3), (1.4) в примере 1.

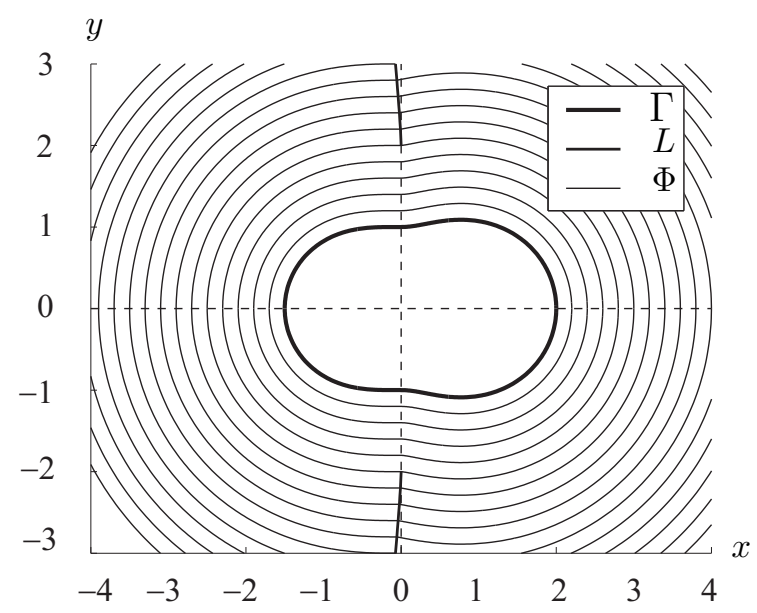

Рис. 2. Линии уровня решения задачи (1.3), (1.4) в примере 2 .

целевым множеством. Его основу составляют разрабатываемые теоретические подходы для выявления сингулярностей, а также методы дифференциальной [17] (отыскание радиуса и центра кривизны) и вычислительной геометрии [20] (нахождение пересечения отрезков и лучей). В первом из рассматриваемых ниже примеров в псевдовершинах целевого множества определен радиус кривизны, а во втором примере классическая кривизна не определена, но существуют односторонние ее пределы слева и справа. Уравнения, задающее кривую Г, имеют в качестве параметра угол радиус-вектора точки, образованный с положительным направлением оси абсцисс. Это позволяет записать выражения (1.2) в наиболее удобном и компактном виде, в нем используются в основном функции синуса и косинуса и их комбинации.

П р и м е р 1. Пусть в задаче (1.3), (1.4) краевое условие задано на границе множества $M$, ограниченного кривой $\Gamma$, описываемой уравнениями

$$
\left\{\begin{array}{l}
x=\left(1.5 \sin ^{2} t+0.5 \cos 2 t\right) \cos t \\
y=\left(1.5 \sin ^{2} t+0.5 \cos 2 t\right) \sin t
\end{array}\right.
$$

где параметр $t$ принимает значения из отрезка $[0, T]=[0,2 \pi]$.

Множество $M$ имеет 4 псевдовершины, в которых кривизна определена и достигает локального максимума. В них выполняются условия теоремы 2 , а значит, имеет место предельное соотношение (2.6). Координаты псевдовершин $\mathbf{y}_{1} \approx(0.4312,0.1921), \mathbf{y}_{2} \approx(-0.4312,0.1921)$, $\mathbf{y}_{3} \approx(-0.4312,-0.1921), \mathbf{y}_{4} \approx(0.4312,-0.1921)$ находятся путем отыскания точек максимума выражения (2.2) как функции от параметра $t$ при его заданных ограничениях, а затем вычисления в них значений (3.1). Координаты соответствующих им крайних точек биссектрисы $\widehat{\mathbf{x}}_{1} \approx(0.5587,0.2138), \widehat{\mathbf{x}}_{2} \approx(-0.5587,0.2138), \widehat{\mathbf{x}}_{3} \approx(-0.5587,-0.2138), \widehat{\mathbf{x}}_{4} \approx(0.5587,-0.2138)$. Построение ветвей сингулярного множество осуществлялось посредством решения задачи Коши (2.7). Биссектриса $L(M)$ состоит из 2 компонент связности, каждая из которых есть объединение 3 одномерных и 1 нульмерного многообразия. Координаты этих нульмерных многообразий (точек бифуркации биссектрисы) $-\overline{\mathbf{x}}_{1} \approx(5.636,0)$ и $\overline{\mathbf{x}}_{2} \approx(-5.636,0)$. Линии уровня $\Phi$ решения задачи с краевым условием (3.1), сингулярное множество $L$ и граница $\Gamma$ целевого множества представлены на рис. 1.

П р и м е р 2. Пусть в задаче (1.3), (1.4) краевое условие определено на границе множества $M$, ограниченного кривой $\Gamma$, заданной уравнениями

$$
x(t)=\left\{\begin{array}{l}
\left(2-\sin ^{2} t\right) \cos t, t \in[0, \pi / 2] \cup[3 \pi / 2,2 \pi], \\
\left(1-0.5 \sin ^{2}(t)\right) \cos t, t \in(\pi / 2,3 \pi / 2),
\end{array}\right.
$$




$$
y(t)=\left\{\begin{array}{l}
\left(2-\sin ^{2} t\right) \sin t, t \in[0, \pi / 2] \cup[3 \pi / 2,2 \pi], \\
\left(1.5-0.5 \sin ^{2} t\right) \sin t, t \in(\pi / 2,3 \pi / 2),
\end{array}\right.
$$

где параметр $t$ принимает значения из отрезка $[0, T]=[0,2 \pi]$.

Множество $M$ имеет 2 псевдовершины, в которых кривизна кривой (3.2), (3.3) не определена, но существуют ее односторонние пределы. В них выполняются условия теоремы 3 , координаты крайних точек кривой (2.1) вычисляются в первом случае по формуле (2.8), а во втором случае по формуле (2.9). Координаты псевдовершин определим как $\mathbf{y}_{1}=(0,1)$ и $\mathbf{y}_{2}=(0,-1)$. Построение ветвей сингулярного множество осуществлялось посредством решения задач Коши (2.22) и (2.23). Биссектриса $L(M)$ состоит из 2 компонентов связности. Крайние точки биссектрисы имеют координаты $\widehat{\mathbf{x}}_{1}=(0,2)$ и $\widehat{\mathbf{x}}_{2}=(0,-2)$. Линии уровня $\Phi$ решения задачи с краевым условием (3.2), (3.3), сингулярное множество $L$ и граница Г целевого множества представлены на рис. 2.

\section{Заключение}

В статье изучены свойства сингулярных множеств в одном классе задач управления по быстродействию с круговой вектограммой скоростей. Систематизированы различные типы характеристических точек границы множества (псевдовершин), найдены выражения для координат крайних точек сингулярных кривых (биссектрис). Получены предельные соотношения для параметров, задающих координаты точек границы целевого множества, в которые приходят оптимальные траектории, исходящие из одной точки. На основе этих соотношений предложены и реализованы процедуры построения рассеивающих линий с помощью решения задач Коши для дифференциального уравнения первого порядка, связывающего параметры точек, порождающих биссектрису множества. Выполнено моделирование решений задач управления для целевых множеств с различными дифференциальными свойствами границы - для случая, когда в точках границы классическая кривизна определена, и для случая, когда существуют точки границы, в которых кривизна разрывна.

\section{СПИСОК ЛИТЕРАТУРЫ}

1. Красовский Н.Н., Субботин А.И. Позиционные дифференциальные игры. М.: Наука, 1974. $456 \mathrm{c}$.

2. Лебедев П.Д., Успенский А.А. Аналитическое и численное конструирование функции оптимального результата для одного класса задач быстродействия// Прикл. математика и информатика: тр. фак-та ВМК Моск. ун-та. 2007. № 27. С. 65-79.

3. Лебедев П.Д., Успенский А. А. Геометрия и асимптотика волновых фронтов// Изв. вузов. Математика. 2008. № 3 (550). С. 27-37.

4. Ушаков В.Н., Успенский А. А., Лебедев П. Д. Геометрия сингулярных кривых для одного класса задач быстродействия // Вестн. С.-Петерб. ун-та. Сер. 10. 2013. Вып. 3. С. 157-167.

5. Успенский А.А., Лебедев П.Д. Построение функции оптимального результата в задаче быстродействия на основе множества симметрии // Автоматика и телемеханика. 2009. № 7. С. 50-57.

6. Lebebev P.D., Uspenskii A.A., Ushakov V.N. Construction of nonsmooth solutions in one class of velocity problems // Constructive Nonsmooth Analysis and Related Topics (dedicated to the memory of V.F. Demyanov), CNSA-2017: Proc. conf. 2017. Article no. 7973981. P. 185-188. doi: 10.1109/CNSA.2017.7973981.

7. Айзекс Р. Дифференциальные игры. Москва: Мир, 1967. 479 с.

8. Субботин А.И. Обобщенные решения уравнений в частных производных первого порядка. Перспективы динамической оптимизации. Москва; Ижевск: Изд-во Ин-та компьютерных технологий, 2003. $336 \mathrm{c.}$

9. Кружков С.Н. Обобщенные решения уравнений Гамильтона - Якоби типа эйконала. Постановка задач, теоремы существования, единственности и устойчивости, некоторые свойства решений. I // Мат. сб. 1975. Т. 98 (140). № 3 (11). С. 450-493. 
10. Демьянов В.Ф., Васильев Л. В. Недифференцируемая оптимизация. Москва: Наука, 1981. $384 \mathrm{c}$.

11. Sedykh V.D. On the topology of cooriented wave fronts in spaces of small dimensions// Mosc. Math. J. 2011. Vol. 11, no. 3. P. 583-598.

12. Седых В.Д. О топологии волновых фронтов в пространствах небольших размерностей// Изв. РАН. Cер. математическая. 2012. Т. 76, вып. 2. С. 171-214. doi: https://doi.org/10.4213/im8202 .

13. Арнольд В.И. Особенности каустик и волновых фронтов. Москва: ФАЗИС, 1996. 334 с.

14. Giblin P.J., Warder J.P. Evolving evolutoids // American Math. Monthly. 2014. Vol. 121, iss. 10. P. 871-889. doi: 10.4169/amer.math.monthly.121.10.871.

15. Giblin P.J., Reeve G. Centre symmetry sets of families of plane curves// Demonstratio Mathematica. 2015. Vol. 48, iss. 2. P. 167-192. doi: 10.1515/dema-201-0016.

16. Местецкий Л. М. Непрерывная морфология бинарных изображений: фигуры, скелеты, циркуляры. Москва: Физматлит, 2009. 288 с.

17. Рашевский П.К. Курс дифференциальной геометрии. Москва:: "Едиториал УРСС", 2003. 432 с.

18. Лейхтвейс К. Выпуклые множества. Москва:: Наука, 1985. 335 с.

19. Лебедев П. Д., Успенский А.А. Программа построения волновых фронтов и функции евклидова расстояния до компактного невыпуклого множества. Свид-во о гос. регистрации программы для ЭВМ № 2017662074 от 27.10.2017.

20. Preparata F.P., Shamos M.I. Computational geometry: An introduction. N Y: Springer-Verlag, 1988.

Поступила 5.12 .2018

После доработки 7.02.2019

Принята к публикации 11.02.2019

Лебедев Павел Дмитриевич

канд. физ.-мат. наук, старший науч. сотрудник

Институт математики и механики им. Н.Н. Красовского УрО РАН;

Уральский федеральный университет

г. Екатеринбург

e-mail: pleb@yandex.ru

Успенский Александр Александрович

д-р. физ.-мат. наук, ведущий науч. сотрудник

Институт математики и механики им. Н.Н. Красовского УрО РАН;

Уральский федеральный университет

г. Екатеринбург

e-mail: uspen@imm.uran.ru

\section{REFERENCES}

1. Krasovskii N.N., Subbotin A.I. Game-theoretical control problems. N Y: Springer, 1988, 517 p. ISBN: 9781-4612-8318-8. Original Russian text published in Krasovskii N.N., Subbotin A.I. Pozitsionnye differentsial'nye igry. Moscow, Nauka Publ., 1974, 456 p.

2. Lebedev P.D., Uspenskii A.A. Analytical and numerical construction of the optimal outcome function for a class of time-optimal problems. Comput. Math. Model., 2008, vol. 19, no. 4, pp. 375-386. doi: 10.1007/s10598-008-9007-9.

3. Lebedev P.D., Uspenskii A.A. Geometry and asymptotics of wavefronts. Russian Mathematics (Izvestiya VUZ. Matematika), 2008, vol. 52, no. 3, pp. 24-33. doi: 10.3103/S1066369X08030031.

4. Ushakov V.N., Uspenskii A.A., Lebedev P.D. Geometry of singular curves for one class of velocity. Vestn. S.-Petersburg Univer. Ser. 10. Prikl. Mat. Inform. Prots. Upr., 2013, no. 3, pp. 157-167 (in Russian).

5. Uspenskii A.A., Lebedev P.D. Construction of the optimal outcome function for a time-optimal problem on the basis of a symmetry set. Autom. Remote Control, 2009, vol. 70, no. 7, pp. 1132-1139. doi: 10.1134/S0005117909070054.

6. Lebebev P.D., Uspenskii A.A., Ushakov V.N. Construction of nonsmooth solutions in one class of velocity problems. Constructive Nonsmooth Analysis and Related Topics (dedicated to the memory of V.F.Demyanov), CNSA 2017 - Proc. 10 July 2017, article no. 7973981, pp. 185-188. doi: 10.1109/CNSA.2017.7973981. 
7. Isaacs R. Differential games. N Y: John Wiley and Sons, 1965, 384 p. ISBN: 0471428604 . Translated to Russian under the title Differentsial'nye igry. Moscow, Mir Publ., 1967, 479 p.

8. Subbotin A.I. Generalized solutions of first-order PDEs. The dynamical optimization perspective. Basel, Birkhäuser, 1995. 314 p. doi: 10.1007/978-1-4612-0847-1. Translated to Russian under the title Obobshchennye resheniya uravnenii $v$ chastnykh proizvodnykh pervogo poryadka: Perspektivy dinamicheskoi optimizatsii. Moscow, Izhevsk: Inst. Komp'yuter. Issled. Publ., 2003. 336 p.

9. Kruzhkov S.N. Generalized solutions of the Hamilton-Jacobi equations of eikonal type. I. Formulation of the problems; existence, uniqueness and stability theorems; some properties of the solutions, Mathematics of the USSR-Sbornik, 1975, vol. 27, no. 3, pp. 406-446. doi: 10.1070/SM1975v027n03ABEH002522 .

10. Dem'yanov V.F., Vasil'ev L.V. Nondifferentiable optimization. N Y: Springer-Verlag, 1985, 452 p. ISBN: 978-0-387-90951-6. Original Russian text published in Dem'yanov V.F., Vasil'ev L.V. Nedifferentsiruemaya optimizatsiya. Moscow: Nauka Publ., 1981, 384 p.

11. Sedykh V.D. On the topology of cooriented wave fronts in spaces of small dimensions. Mosc. Math. J., 2011, vol. 11, no. 3, pp. 583-598.

12. Sedykh,Vyacheslav D. On the topology of wave fronts in spaces of low dimension. Izvestiya: Mathematics, 2012, vol. 76, no. 2, pp. 375-418. doi: 10.1070/IM2012v076n02ABEH002588.

13. Arnold V.I. Singularities of caustics and wave fronts. Springer Netherlands, 1990, Math. Appl., vol. 62, 259 p. doi: 10.1007/978-94-011-3330-2. Translated to Russian under the title Osobennosti kaustik $i$ volnovyh frontov. Moscow: FAZIS Publ., 1996, 334 p.

14. Giblin P.J., Warder J.P. Evolving evolutoids. American Mathematical Monthly, 2014, vol. 121, no. 10, pp. 871-889. doi: 10.4169/amer.math.monthly.121.10.871.

15. Giblin P.J., Reeve G. Centre symmetry sets of families of plane curves. Demonstratio Mathematica, 2015, vol. 48, no. 2, pp. 167-192. doi: 10.1515/dema-201-0016.

16. Mestetskiy L.M. Nepreryvnaja morfologija binarnyh izobrazhenij: figury, skelety, cirkuljary (Continuous morphology of binary images. Figures, skeletons, circular). Moscow: Fizmatlit Publ., 2009, 288 p. ISBN: 978-5-9221-1050-1.

17. Rashevskii P.K. Kurs differencial'noj geometrii [A Course in Differential Geometry]. Moscow: URSS Publ., 2003, 432 p. ISBN: 5-354-00294-X.

18. Leichtweiss K. Konvexe Mengen. Berlin: Springer, 1980, 330 p. ISBN: 978-3-540-09071-7. Translated to Russian under the title Vypuklye mnozhestva. Moscow: Nauka Publ., 1985, 335 p.

19. Lebedev P.D., Uspenskii A.A. Program for constructing wave fronts and functions of the Euclidean distance to a compact nonconvex set. Certificate of state registration of the computer program no. 2017662074, October 27, 2017.

20. Preparata F.P., Shamos M.I. Computational geometry: An introduction. N Y: Springer-Verlag, 1988, 400 p. doi: $10.1007 / 978-1-4612-1098-6$.

Received December 5, 2018

Revised February 7, 2019

Accepted February 11, 2019

Funding Agency: This work was supported by Resolution No. 211 of March 16, 2013, of the Government of the Russian Federation (agreement no. 02.A03.21.0006).

Pavel Dmitrievich Lebedev, Cand. Sci. (Phys.-Math.), Krasovskii Institute of Mathematics and Mechanics of the Ural Branch of the Russian Academy of Sciences, Yekaterinburg, 620108 Russia; Ural Federal University, Yekaterinburg, 620002 Russia, e-mail: pleb@yandex.ru .

Aleksandr Aleksandrovich Uspenskii, Dr. Phys.-Math. Sci., Krasovskii Institute of Mathematics and Mechanics of the Ural Branch of the Russian Academy of Sciences, Yekaterinburg, 620108 Russia; Ural Federal University, Yekaterinburg, 620002 Russia, e-mail: uspen@imm.uran.ru . 